\title{
BMJ Open Identification of the optimal points for the acupuncture treatment of neck pain in China: protocol for a multicenter, matched, case-control study
}

\author{
Mingsheng Sun, ${ }^{\oplus 1}$ Siyuan Tao, ${ }^{2}$ Guoyan Geng, ${ }^{1}$ Jieru Peng, ${ }^{2}$ Xingsha Ma, ${ }^{1}$ \\ Mingxi Yan, ${ }^{1}$ Jiao Chen, ${ }^{1}$ Dingjun Cai, ${ }^{1}$ Hui Zheng, ${ }^{1}$ Chunxia Yang, ${ }^{2}$ Ling Zhao, ${ }^{1}$ \\ Fan-rong Liang ${ }^{1}$
}

To cite: Sun M, Tao S, Geng G, et al. Identification of the optimal points for the acupuncture treatment of neck pain in China: protocol for a multicenter, matched, case-control study. BMJ Open 2019;9:e029194. doi:10.1136/ bmjopen-2019-029194

- Prepublication history for this paper is available online. To view these files please visit the journal online (http://dx.doi org/10.1136/bmjopen-2017016527).

MS, ST and GG contributed equally.

Received 16 January 2019

Revised 19 July 2019

Accepted 23 July 2019

D) Check for updates

(c) Author(s) (or their employer(s)) 2019. Re-use permitted under CC BY-NC. No commercial re-use. See rights and permissions. Published by BMJ.

${ }^{1}$ College of Acupuncture and Tuina, Chengdu University of Traditional Chinese Medicine, Chengdu, China

${ }^{2}$ West China School of Public Health, Sichuan University, Chengdu, China

Correspondence to Professor Fan-rong Liang; acuresearch@126.com

Professor Ling Zhao; zhaoling@cdutcm.edu.cn

\section{ABSTRACT}

Introduction Neck pain (NP) is a common condition that can be effectively treated by acupuncture. However, several treatment point prescriptions (ie, local acupoints, distal acupoints, and sensitised acupoints) may be used. The present study aims to identify the types of sensitisation and the distribution of sensitised points in patients with NP, to analyse the cut-off values and sensitisation rate for acupoint sensitisation, and to summarise the dominant forms of optimally sensitised points. This information will be helpful when choosing the optimal points to treat NP.

Methods and analysis This multicentre, matched, case-control study will enrol 224 patients with NP, and 224 age-matched and sex-matched healthy participants as controls. Body surface temperature, mechanical pain threshold, pressure pain threshold and skin resistance will be assessed at the 15 acupoints most frequently used to treat NP, and at the five body regions in which pain occurs most frequently. Hypothesis testing will be used to compare the differences in variables between cases and controls. In addition, receiver operating characteristic curve analysis will be used to explore the cut-off values of the sensitive states of heat, pain and electrical resistance, which indicate sensitisation of the acupoint. The optimal points will be comprehensively determined by the acupoint sensitisation rate and $\mathrm{OR}$.

Ethics and dissemination Ethical approval of this study has been granted by the Research Ethical Committee of the Teaching Hospital of Chengdu University of Traditional Chinese Medicine (ID: 2018 KL-016). The outcomes of the study will be disseminated through peer-reviewed publications.

Trial registration ChiCTR1800016220.

\section{INTRODUCTION}

Neck pain (NP) is a common clinical condition often accompanied by tenderness at sensitive points. The global lifetime prevalence of NP was $48.5 \%$ in $2006 .{ }^{1}$ The prevalence in white-collar workers in China was $33.9 \%-54.8 \%$ in 2016 and has increased in recent years, ${ }^{2}$ imposing considerable
Strengths and limitations of this study

- This study will provide information about the forms of sensitisation and the distribution of sensitised points in patients with neck pain (NP) using the largest known sample, in a strict observational study.

- Objective or semiobjective biological measure, body surface temperature, mechanical pain threshold, pressure pain threshold and skin resistance will be assessed as a preliminarily exploration of the different forms of sensitisation.

- This study will attempt to define acupoint sensitisation in patients with NP using a cut-off value for identifying point sensitisation.

- The representativeness and generalisability of the study results will be limited because we are including only patients with non-traumatic NP with mobility deficits.

personal and socioeconomic burdens. Muscle relaxants and non-steroidal anti-inflammatory drugs are used to treat this condition; however, these medications carry a risk of adverse effects, and neither drug is better than non-pharmacological alternative treatments. ${ }^{1}$

Because non-pharmacological alternative treatments have reliable efficacy and availability, this type of treatment is gaining increasing recognition worldwide. Acupuncture is one type of non-pharmacological alternative treatment that can effectively treat NP. Although several studies have confirmed that acupuncture is effective, outcomes are closely related to point selection. ${ }^{3}$ Previous studies have evaluated the use of local acupoints ${ }^{4}$ or distal acupoints, ${ }^{5}$ with the treatment of both types reportedly having a certain degree of clinical efficacy.

Recent Chinese research has found that acupoints can be sensitised, with associated 
changes in function and size, and thus can functionally change from a relatively 'silent' state to a relatively 'active' state. Treatment of an acupoint while it is in the 'active' state is thought to achieve a better clinical outcome, and this has become a focus in acupoint research. ${ }^{6-8}$ Several types of sensitivity manifest at sensitised points, including pain sensitivity, heat sensitivity and form sensitivity (appearance changes). Recent clinical studies report that a superior outcome is obtained using acupuncture at pain-sensitive points, ${ }^{9}$ and moxibustion at heat-sensitive points ${ }^{10}$ or visually sensitive points. ${ }^{11}$ However, most of the current observational studies have focused on only one form of sensitisation in small-sized sample populations, and thus are not comprehensive studies of different forms of sensitisation. ${ }^{610}$ The choice of optimal treatment points for NP in clinical practice remain controversial.

Herein, we describe the protocol for an observational study that aims to identify the different types of sensitisation (eg, temperature, tenderness) and the distribution of sensitised acupoints in patients with NP to analyse the cut-off values for acupoint sensitisation, and to determine the most optimal sensitisation types seen at sensitised points. We believe that in the state of disease, sensitive points occur with high frequency (manifested as changes in temperature, pain threshold etc.), that sensitive points with different sensitisation overlap and that the optimal overlapping points may be closely related to selecting the clinical treatment points. Previous small samplesized studies have confirmed the feasibility of sensitisation testing. ${ }^{7}$ The present study will provide evidence for selecting the optimal treatment points for NP in clinical practice.

\section{METHODS AND ANALYSIS}

\section{Study design}

This is a multicentre, age-matched and sex-matched casecontrol study. The protocol was developed in accordance with the Strengthening the Reporting of Observational Studies in Epidemiology guidelines. ${ }^{12}$ The study has been registered with ChiCTR at Current Controlled Trials (ChiCTR1800016220). A flowchart of the study design is shown in figure 1.

\section{Ethics}

This study was designed in accordance with the principles of the Declaration of Helsinki. The study protocol has been approved, and is registered on the primary registry in the WHO registry network (Chinese Clinical Trial Registry: no. ChiCTR1800016220). Signed consent will be obtained from each participant after they have been informed of the study procedures, possible risks and their right to withdraw from the study.

\section{Patients and healthy participants (controls) Inclusion criteria \\ Patients are eligible for study inclusion if they: (1) have non-traumatic NP with mobility deficits in the acute and}

chronic stages, (2) are men or women aged 18-60 years and (3) provide written informed consent for all procedures in this study.

Healthy participants as controls subjects are eligible if they: (1) have no history of NP and/or restricted neck movement, (2) are men or women aged 18-60 years and (3) provide written informed consent for all procedures in this study.

\section{Exclusion criteria}

Patients are ineligible if they: (1) have complicated neck or shoulder pain caused by cervical and intervertebral disc degeneration, such as other types of cervical spondylosis, shoulder periarthritis, rheumatic myofibrillar inflammation; (2) have history of neck fracture or surgery or cervical congenital abnormality; (3) have serious disease related to the heart, liver, kidney or hematopoietic system; (4) have difficulty answering questionnaires because of cognitive impairment; (5) have dermatopathological diseases; or (6) are pregnant, breastfeeding or planning a pregnancy during the study period.

Healthy participants are ineligible if they: (1) have serious disease related to the heart, liver, kidney or hematopoietic system; (2) have cervical congenital abnormality; (3) have difficulty answering the questionnaires because of cognitive impairment; (4) have dermatopathological diseases; or (5) are pregnant, breastfeeding or planning a pregnancy during the study period.

\section{Recruitment strategies}

We will recruit patients from the outpatient departments of Acupuncture and Moxibustion, and Orthopaedics in five clinical centres in China: Chengdu University of Traditional Chinese Medicine, Hunan University of Traditional Chinese Medicine, Shaanxi University of Traditional Chinese Medicine, Shanxi University of Traditional Chinese Medicine and Guiyang College of Traditional Chinese Medicine. Healthy participants without NP or shoulder pain will comprise age-matched and sex-matched residents from the same communities as the patients, to act as controls. Recruitment strategies will include posting advertisements on social media (such as WeChat, which is similar to Facebook) and at community centres, or by word-of-mouth introductions through participants already enrolled in the study. Patients or healthy participants who consent to study participation will be examined and diagnosed by a hospital doctor.

\section{Test regions, acupoints and sensitised points}

Following the results of literature data-mining and expert consensus on the treatment of NP, we identified the 15 most frequently used acupoints (table 1) and the five regions of the body with the most frequent occurrence of pain and the greatest degree of acupoint sensitisation. The body will be divided into five regions to standardise the treatment procedures and detection areas. Regions 1 and 2 are each bordered by the respective ipsilateral mastoid, sternal end of the clavicle, anterior axillary 

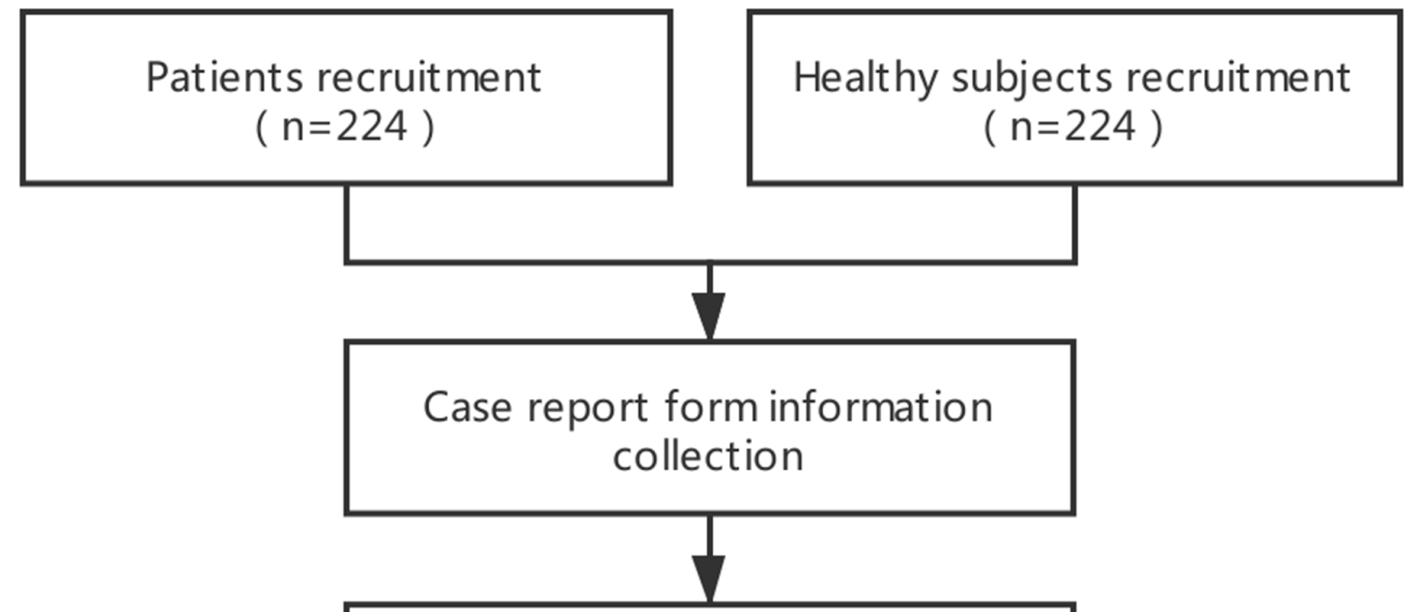

Measurements of body surface temperature

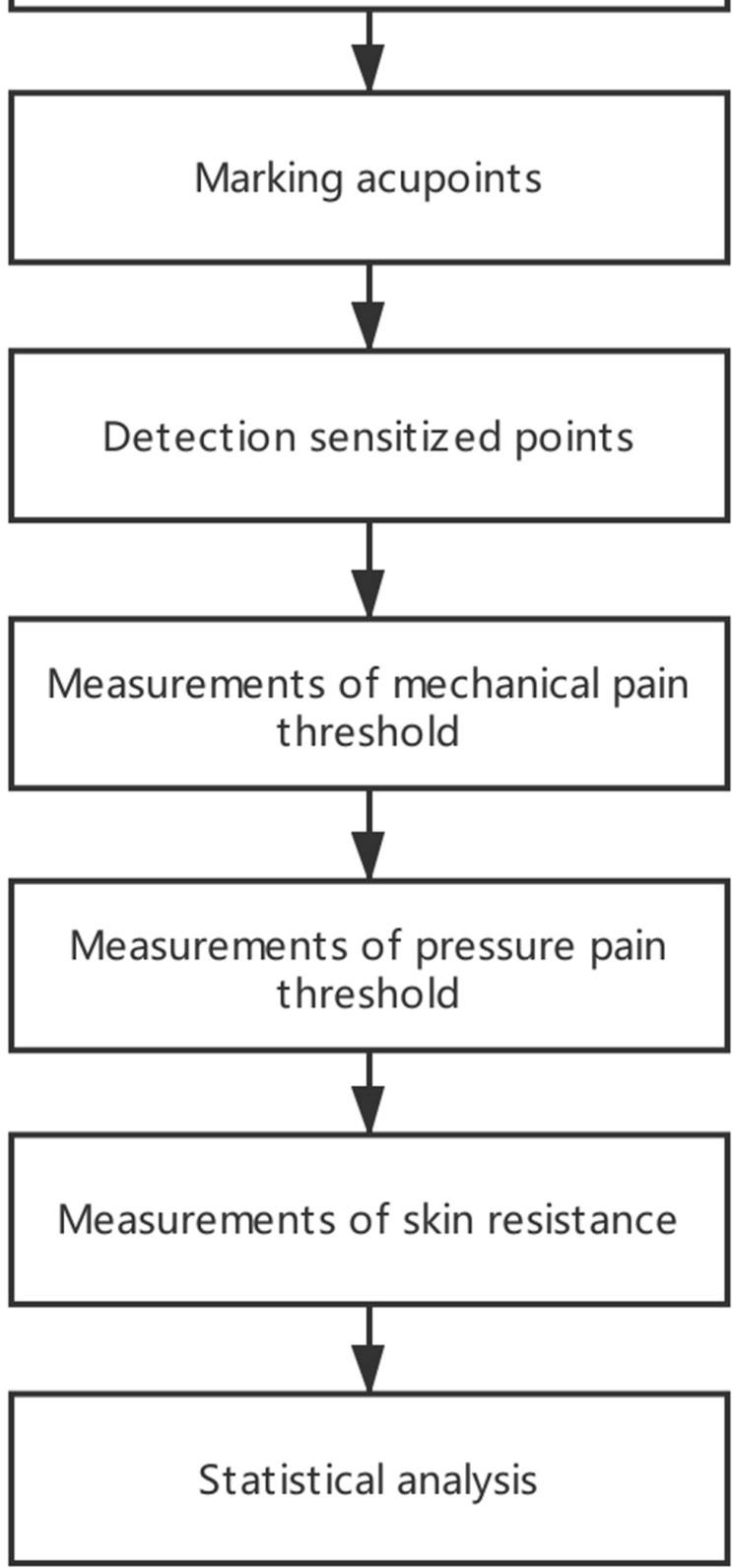

Figure 1 Flowchart of the study design. 
Table 1 Acupoints selected for use in the study

\begin{tabular}{|c|c|}
\hline Acupoints & Location \\
\hline Jianjing (GB-21) & $\begin{array}{l}\text { On the shoulder, directly above the nipple, at the midpoint of the line connecting Dazhui (DU-14) with the } \\
\text { acromial end of clavicle }\end{array}$ \\
\hline $\begin{array}{l}\text { Jianzhongshu } \\
(\mathrm{SI}-15)\end{array}$ & On the back, 2 cun lateral to the lower border of the spinous process of the seventh cervical vertebra \\
\hline Wangu (GB-12) & On the head, in the depression posterior and inferior to the mastoid process. \\
\hline Fengchi (GB-20) & $\begin{array}{l}\text { On the nape, below the occipital, on a level with Fengfu (DU-16), in the depression between the upper } \\
\text { portion of trapezius and the sternocleidomastoid }\end{array}$ \\
\hline Tianzhu (BL-10) & $\begin{array}{l}\text { On the nape, } 1.3 \text { cun lateral to the posterior hairline, in the depression of the posterior hairline lateral to the } \\
\text { trapezius muscle }\end{array}$ \\
\hline Dazhui (DU-14) & On the posterior median line, in the depression below the spinous process of the seventh cervical vertebra \\
\hline Dazhu (BL-11) & On the back, 1.5 cun lateral to the lower border of the spinous process of the first thoracic vertebra \\
\hline Jianwaishu (SI-14) & On the back, 3 cun lateral to the lower border of the spinous process of the first thoracic vertebra \\
\hline Tianliao (SJ-15) & $\begin{array}{l}\text { On the region of scapula, at the midpoint of the line connecting Jianjing (GB-21) with Quyuan (SI-13), on } \\
\text { the superior angle of the scapula }\end{array}$ \\
\hline Jugu (LI-16) & $\begin{array}{l}\text { In the upper portion of the shoulder, in the depression between the acromial end of clavicle and the } \\
\text { scapular spine }\end{array}$ \\
\hline Tianzong (SI-11) & $\begin{array}{l}\text { In the region of the scapula, in the depression of the centre of the subscapular fossa, on a level with the } \\
\text { fourth thoracic vertebra }\end{array}$ \\
\hline Shousanli (LI-10) & $\begin{array}{l}\text { Flexing the elbow, on the dorsal radial side of the forearm, on the line connecting Yangxi (LI-5) with Quchi } \\
\text { (LI-11), } 2 \text { cun below the transverse cudital crease }\end{array}$ \\
\hline Lieque (LU-7) & $\begin{array}{l}\text { On the radial margin of the forearm, } 1.5 \text { cun above the transverse crease of the wrist, between the } \\
\text { branchioradial muscle and the long abductor muscle tendon of thumb }\end{array}$ \\
\hline Zhongzhu (SJ-3) & $\begin{array}{l}\text { On the dorsum of the hand, in the depression between the fourth and fifth metacarpal bones, proximal to } \\
\text { the fourth metacarpalangeal joint }\end{array}$ \\
\hline Houxi (SI-3) & $\begin{array}{l}\text { On the ulna side of the palm, proximate to the fifth metacarpophalangeal joint, at the end of transverse } \\
\text { crease of metacarpophalangeal joint, at the dorsoventral boundary }\end{array}$ \\
\hline
\end{tabular}

line, acromion and C7 spinous process. Region 3 is the triangular region bordered by both sides of the mastoid and the $\mathrm{C} 7$ spinous process. Regions 4 and 5 are each bordered by the respective ipsilateral $\mathrm{C} 7$ spinous process, acromion and axillary line; the two regions are divided by the posterior midline. These regions are the same as those used in previous studies of $\mathrm{NP},{ }^{13}$ and include the upper trapezius, splenius capitis muscles, sternocleidomastoid, levator scapulae, infraspinatus, scalene, subscapularis muscles and other neck muscles associated with NP. ${ }^{1415}$ These regions and acupoints are shown in figure 2.

Investigators will mark the 29 acupoints on each patient. All investigators will attend training to ensure all practices are standardised. Investigators will be blinded regarding whether participants are patients or healthy controls. The investigator will palpate the detection area associated with each acupoint using the appropriate force $(<2000 \mathrm{gf})$, and will identify sensitised points that have pain/sourness/heaviness/fullness or nodules.

\section{Outcome measurements}

All data collection for each patient will be completed on the same day.

\section{Body surface temperature}

Thermal imaging technology has been widely used to assess pain and muscle damage. ${ }^{16}{ }^{17}$ Each participant will be evaluated in a room with a constant temperature of $26^{\circ} \mathrm{C}$. Investigators will use thermal imaging cameras

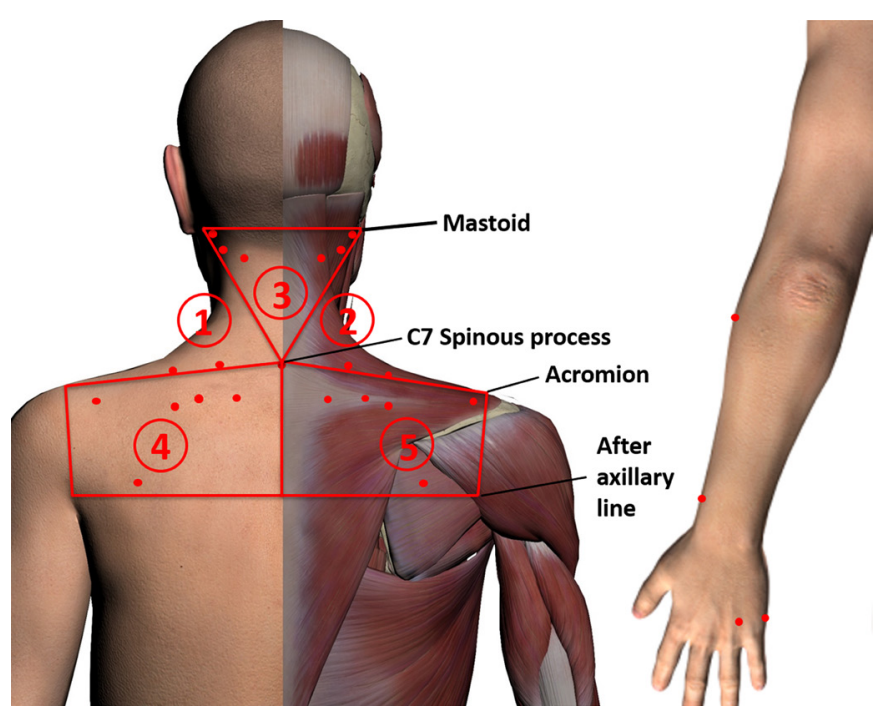

Figure 2 The test regions and acupoints that will be used in the study. 
(Fotric 226, IRS Systems, Allen, Texas, USA) to take measurements at each of the 29 acupoints in the five body regions. The temperature data for each point on the images will be analysed using professional software (AnalyzIR, IRS Systems, Allen, Texas, USA).

\section{Mechanical pain threshold}

Investigators will use the electronic Von Frey esthesiometer (model 2390; IITC Life Science, Woodland Hills, California, USA) to take two measurements of the mechanical pain threshold at each of the 29 acupoints in the five body regions in turn. Previous studies have found that this device can detect hyperalgesia and changes in sensory nerve function. ${ }^{18-20}$ Mechanical pain caused by punctate stimuli is related to $A \delta$-fibres. ${ }^{21}$ If there is a difference of more than $15 \mathrm{~g}$ between the two measurements of mechanical pain threshold made at one acupoint, the mechanical pain threshold at that acupoint will be measured a third time. Progressive pressure will be applied at a rate of $10 \mathrm{~g} / \mathrm{s}$ at each acupoint, and we will calculate the average mechanical pain threshold for each acupoint. To reduce the consecutive assessment error for two adjacent points, an alternate assessment method for acupoints on the left and right sides of the body will be adopted.

\section{Pressure pain threshold}

The pressure pain threshold (PPT) is widely used clinically as a semiobjective method with which to quantify localised pain. ${ }^{22} 23$ Pain caused by blunt pressure stimuli is related to C-fibres. ${ }^{21}$ Investigators will use the FDIX Force Gauge (Force One FDIX, Wagner Instruments, Greenwich, Connecticut, USA) to take two PPT measurements at each of the 29 acupoints in the five body regions in turn. If there is a difference of more than 500 gf between the two PPT measurements performed at one acupoint, the PPT at that acupoint will be measured a third time. Progressive pressure will be applied at a rate of $100 \mathrm{gf} / \mathrm{s}$ at each acupoint, and the average PPT will be calculated. An alternate assessment method for the acupoints on the left and right sides of the body will be adopted.

\section{Skin resistance}

Investigators will use the Acupoint Dynamics Research Instrument (LMR30-RIII, Peking University, Beijing, China) to record the skin resistance at nine acupoints, bilateral (GB-21, GB-20, BL-11, LI-10, SI-3) and at the two most sensitive points (if there are two sensitive points).

\section{Pain}

NP will be measured using the Visual Analogue Scale, the Northwick Park Neck Pain Questionnaire and the McGill Pain Questionnaire. The visual analogue scale will be used to measure pain severity. The Northwick Park Neck Pain Questionnaire provides a reliable outcome measure for patients with NP, and the McGill Pain Questionnaire is used to measure the different qualities of subjective pain; these questionnaires have been proven valid, and are convenient for patients to complete. ${ }^{24} 25$

\section{Neck function}

Changes in neck function will be evaluated by measuring cervical range of motion.

\section{General demographic information}

This will include the collection of data regarding age, disease duration, medical history and medication type and dosage.

\section{Safety and adverse events}

Non-invasive assessments of the body surface do not generally cause adverse events. However, we will record the number and type (eg, serious pain, fainting) of adverse events in each group. Participants who experience adverse events will receive the appropriate intervention. We will report adverse events immediately to the primary investigator and the ethics committee, and the affected participants will be withdrawn from the study.

\section{Patients and public involvement}

Patients and the public have not and will not be involved in the design and conception of this study.

\section{Sample size calculation}

A previous study indicated that the rate of acupoint sensitisation in patients ranged from $20 \%$ to $70 \%^{26}$; therefore, we set this rate at $50 \%$ to calculate the minimum sample size required for the proposed study. With an acupoint sensitisation rate of $20 \%$ in healthy participants, the OR is 4 . According to Chow's formula for comparing two sample proportions, ${ }^{27}$ we assumed $\alpha=0.05$, and $\beta=0.01$; thus, the smallest sample size is 408 with two-sided confidence and a ratio of control participants to cases of 1 (TrialSize package in R; The R Project for Statistical Computing, Copenhagen, Denmark). Setting the potential non-response rate and sampling effectiveness at $10 \%$, the final sample size is 448 (224 patients, plus 224 age-matched and sex-matched healthy controls).

\section{Statistical analysis}

Data will be blinded, double-entered in EpiData V.3.1 software (EpiData Association, Odense, Denmark), and adequately checked for errors. SPSS V21.0 (IBM Inc, Armonk, New York, USA) will then be used to complete the statistical analyses. The statistical data evaluation will be performed independently by the West China School of Public Health at Sichuan University, China. Missing data will be processed without imputation. First, the distribution of basic information in the case and control groups will be described for age, sex, height, weight, occupation and education level. Data will be presented as means ( $\mathrm{SD}$ ) for continuous variables, and as frequency (percentage) for categorical variables. The distributions of sensitised points will be shown in scatter plots to represent skin morphological changes. We will also describe the distributions of the intensity of NP and neck function. Hypothetical testing will then be performed to assess the differences between the case and control groups regarding body surface sensations and biophysical 
properties, including body surface temperature, mechanical pain threshold, PPT and skin resistance. Parametric statistical testing (t-test) will be used for data that are normally distributed; otherwise, non-parametric statistical testing (Wilcoxon's signed rank test) will be used. If there is a difference between the case and control groups in body surface temperature, mechanical pain threshold, PPT or skin resistance, the cut-off values for these indices to distinguish between sensitive and non-sensitive states (including heat sensitivity, pain sensitivity and electrical sensitivity) will be detected using receiver operating characteristic curve analyses. Finally, the acupoint sensitisation rates of the patients and healthy controls will be calculated separately for heat, pain and electrical sensitivity to identify the most important form of sensitivity for all acupoints. The OR will represent the ratio of acupoint sensitisation in the patients and healthy controls; therefore, we will combine OR and the sensitisation rate of all acupoints to determine the optimal points for each sensitisation.

\section{Quality control}

This is a multicentre observational study; therefore, quality control will play a vital role in extrapolating the conclusions. To ensure the integrity of the study and to protect the rights and health of the participants, we will set up a Data and Safety Monitoring Board. The Data and Safety Monitoring Board will be developed in accordance with the Operational Guidelines for the Establishment and Functioning of the Data and Safety Monitoring Boards of the WHO. Separate from the Data and Safety Monitoring Board, a quality control group will be established to guarantee the validity and reliability of results. Before study commencement, fieldwork operation manuals will be prepared, and pilot study participants from all centres will undergo training in accordance with the standard operating procedure of this study. Every 3 months, members of the quality control group will perform a quality control review at each study site and produce a report regarding the quality analysis of the entire data collection process.

\section{DISCUSSION}

NP is the third -most common chronic pain condition, and the fourth leading cause of disability worldwide. ${ }^{28}$ Acupuncture is a popular non-pharmacological modality to relieve pain. The latest research has reported that acupuncture at sensitive points may provide the most effective treatment. ${ }^{89}$ When the body is in a diseased state, there are morphological form-sensitive point changes, such as nodules, pimples, uplifting, dimpling and changes in skin colour. ${ }^{30}$ Thermal imaging has proven that the temperature of corresponding acupoints will be obviously abnormal when a patient has visceral illness, such as diseases of the heart, lung and stomach, indicating that the temperature of acupoints can reflect the physiological and pathological phenomena of the affected organs. ${ }^{31}$ Studies have confirmed that the PPT at acupoints changes when patients are in a diseased state, ${ }^{6233}$ and that the degree of change in the PPT may reflect the intensity of acupoint sensitisation, and may be related to the disease status. ${ }^{34}$ This proposed observational study will increase knowledge of the different types of acupoint sensitisation and the cut-off values for this sensitisation in patients with NP.

As this will be the first study, to our knowledge, to evaluate the association between acupoint sensitisation and NP, this observational study may have some limitations. The main limitation is that in order to reduce the bias caused by other factors and to ensure consistency, this study will include only patients with non-traumatic NP with mobility deficits, and will exclude patients with other types of NP (such as radiating neck pain or secondary NP). Furthermore, because the four participating centres are located in four different regions in China, it may be difficult to implement quality control; thus, quality control reviews will be performed every 3 months.

In conclusion, this article describes the design and protocol of a study that aims to observe the different types of sensitisation (eg, temperature, tenderness) and the distribution of sensitised acupoints in patients with $\mathrm{NP}$, to analyse the cut-off values for acupoint sensitisation and to identify the dominant sensitised forms of the sensitised points. The results will provide a basis for selecting clinically optimal acupuncture points for NP.

Acknowledgements We thank the staff of Chengdu CTC Tianfu Digital Technology Inc. for providing images. We acknowledge the help and contributions from the subjects, investigators, and experts in each center. We thank Jane Charbonneau, DVM, from Liwen Bianji, Edanz Group China (www.liwenbianji.cn/ac), for editing the English text of a draft of this manuscript.

Contributors MS, ST, JP, DC, CY, HZ, LZ and FL participated in the design of the trial, in creating the data analysis plan, and in drafting the manuscript. GG, XM, MY, and $\mathrm{JC}$ collected the information needed for the performance of this trial in each center. All of the authors discussed, read, and revised the manuscript, and gave final approval for the publication of this study protocol.

Funding This work was financially supported by the National Natural Science Foundation of China (numbers 81590951, 81722050).

Competing interests None declared.

Patient consent for publication Not required.

Ethics approval The study protocol has been approved by the institutional review board and ethics committee of the First Affiliated Hospital of Chengdu University of Traditional Chinese Medicine (May 2018) (approval number: 2018 KL-016).

Provenance and peer review Not commissioned; externally peer reviewed.

Open access This is an open access article distributed in accordance with the Creative Commons Attribution Non Commercial (CC BY-NC 4.0) license, which permits others to distribute, remix, adapt, build upon this work non-commercially, and license their derivative works on different terms, provided the original work is properly cited, appropriate credit is given, any changes made indicated, and the use is non-commercial. See: http://creativecommons.org/licenses/by-nc/4.0/.

\section{REFERENCES}

1. Fejer R, Kyvik KO, Hartvigsen J. The prevalence of neck pain in the world population: a systematic critical review of the literature. Eur Spine J 2006;15:834-48.

2. Guidelines for grading diagnosis and treatment of cervical spondylosis in Anhui Province, China (安徽省颈椎病分级诊疗指南). Anhui Medical Journal 2017;38. 
3. White AR, Ernst E. A systematic review of randomized controlled trials of acupuncture for neck pain. Rheumatology 1999;38:143-7.

4. Mejuto-Vázquez MJ, Salom-Moreno J, Ortega-Santiago R, et al. Short-Term changes in neck pain, widespread pressure pain sensitivity, and cervical range of motion after the application of trigger point dry needling in patients with acute mechanical neck pain: a randomized clinical trial. J Orthop Sports Phys Ther 2014;44:252-60.

5. Irnich D, Behrens N, Gleditsch JM, et al. Immediate effects of dry needling and acupuncture at distant points in chronic neck pain: results of a randomized, double-blind, sham-controlled crossover trial. Pain 2002;99:83-9.

6. Yan C-Q, Zhang S, Li Q-Q, et al. Detection of peripheral and central sensitisation at acupoints in patients with unilateral shoulder pain in Beijing: a cross-sectional matched case-control study. BMJ Open 2017;7:e014438.

7. Luo Y-N, Zhou Y-M, Zhong X, et al. Observation of pain-sensitive points in patients with knee osteoarthritis: a pilot study. Eur $\mathrm{J}$ Integr Med 2018;21:77-81.

8. Chen RX, Kang MF. Acupoint heat-sensitization and its clinical significance. World J Tradit Chin Med 2001;2006:488-9.

9. Yao ZL, Liu F, Song NC. 40 Cases of Neck Type of Cervical Spondylosis Treated by Acupotomy Based on the Theory of Trigger point(基于激痛点理论应用针刀治疗颈型颈椎病40例). Zhongguo zhen $j i u=$ Chinese acupuncture \& moxibustion 2016;36:1155-55.

10. Zhang $\mathrm{H}, \mathrm{Xu} \mathrm{N}$, Huang $\mathrm{R}$, et al. Heat-Sensitive moxibustion in treatment of cervical spondylosis. Liaoning Journal of Traditional Chinese Medicine 2016;12:2631-4.

11. Xiao L, Meng X, Du S, et al. Therapeutic observation of Heavy-dose moxibustion at Sha-petechiae for Neck-type cervical spondylosis due to cold coagulation and blood Stagnation. Shanghai Journal of Acupuncture and Moxibustion 2017;11:1336-9.

12. von Elm E, Altman DG, Egger M, et al. Strengthening the reporting of observational studies in epidemiology (STROBE) statement: guidelines for reporting observational studies. BMJ 2007;335:806-8.

13. Croft PR, Lewis M, Papageorgiou AC, et al. Risk factors for neck pain: a longitudinal study in the general population. Pain 2001;93:317-25.

14. Falla $\mathrm{D}$, Lindstrøm $\mathrm{R}$, Rechter $\mathrm{L}$, et al. Effectiveness of an 8-week exercise programme on pain and specificity of neck muscle activity in patients with chronic neck pain: a randomized controlled study. European Journal of Pain 2013:41:n/a-28.

15. Cagnie B, Castelein B, Pollie F, et al. Evidence for the use of ischemic compression and dry Needling in the management of trigger points of the upper trapezius in patients with neck pain: a systematic review. Am J Phys Med Rehabil 2015;94:573-83.

16. Ring EFJ, Ammer K. Infrared thermal imaging in medicine. Physiol Meas 2012;33:R33-46.

17. Adamczyk JG, Krasowska I, Boguszewski D, et al. The use of thermal imaging to assess the effectiveness of ice massage and cold-water immersion as methods for supporting post-exercise recovery. J Therm Biol 2016;60:20-5.
18. Neto MLP, Maciel LYS, Cruz KML, et al. Does electrode placement influence tens-induced antihyperalgesia in experimental inflammatory pain model? Braz J Phys Ther 2017;21:92-9.

19. Yamamoto $\mathrm{S}$, Ono $\mathrm{H}$, Kume $\mathrm{K}$, et al. Oxaliplatin treatment changes the function of sensory nerves in rats. $J$ Pharmacol Sci 2016;130:189-93.

20. Chen Y, Yao Y, Wu Y, et al. Transcutaneous electric acupoint stimulation alleviates remifentanil-induced hyperalgesia in patients undergoing thyroidectomy: a randomized controlled trial. Int J Clin Exp Med 2015;8:5781-7.

21. Beissner F, Brandau A, Henke C, et al. Quick discrimination of $A$ (delta) and $C$ fiber mediated pain based on three verbal descriptors. PLoS One 2010;5:e12944.

22. Persson AL, Brogårdh $\mathrm{C}$, Sjölund BH. Tender or not tender: testretest repeatability of pressure pain thresholds in the trapezius and deltoid muscles of healthy women. $J$ Rehabil Med 2004;36:17-27.

23. Fischer AA. Pressure algometry over normal muscles. standard values, validity and reproducibility of pressure threshold. Pain 1987;30:115-26.

24. Chiu TT, Lam TH, Hedley AJ. Subjective health measure used on Chinese patients with neck pain in Hong Kong. Spine 2001;26:1884-9.

25. Burckhardt CS, Jones KD. Adult measures of pain: the McGill pain questionnaire (MPQ), rheumatoid arthritis pain scale (RAPS), Short-Form McGill pain questionnaire (SF-MPQ), verbal descriptive scale (VDS), visual analog scale (vas), and West Haven-Yale multidisciplinary pain. Arthritis Care \& Research 2010;49.

26. Chen R, Kang M. Acupoint Heat-Sensitization and its clinical significance. Journal of Traditional Chinese Medicine 2001;2006:488-9.

27. Chow SC, Shao J, Wang H. Sample size calculations in clinical research. New York: Marcel Dekker, 2003.

28. Hoy D, March L, Brooks P, et al. The global burden of low back pain: estimates from the global burden of disease 2010 study. Ann Rheum Dis 2014;73:968-74.

29. Zhang S, Wang W, Zou L, et al. [Acupoints heat-sensitive moxibustion in the application of traditional Chinese surgery]. Zhongguo Zhen Jiu 2016;36:109-12.

30. Zhou S, Yin H, Huai M. Progress and prospect of the study on the correlation mechanism of surface and visceral phase. Acupuncture Research 1982;02.

31. Shui YL. Institute $Q H$, Pasadena. infrared imaging system as a versatile diagnostic and auxiliary treatment tool in Chinese medicine.

32. Chen S, Miao Y, Nan Y, et al. The Study of Dynamic Characteristic of Acupoints Based on the Primary Dysmenorrhea Patients with the Tenderness Reflection on Diji (SP 8). Evid Based Complement Alternat Med 2015;2015:1-9.

33. Chae Y, Kim H-Y, Lee H-J, et al. The alteration of pain sensitivity at disease-specific acupuncture points in premenstrual syndrome. $J$ Physiol Sci 2007;57:115-9.

34. Tunks E, Crook J, Norman G, et al. Tender points in fibromyalgia. Pain 1988;34:11-19. 